\title{
Taming the Goldstone contributions to the effective potential
}

\author{
Stephen P. Martin \\ Department of Physics, Northern Illinois University, DeKalb IL 60115, and \\ Fermi National Accelerator Laboratory, P.O. Box 500, Batavia IL 60510
}

\begin{abstract}
The standard perturbative effective potential suffers from two related problems of principle involving the field-dependent Goldstone boson squared mass, $G$. First, in general $G$ can be negative, and it actually is negative in the Standard Model; this leads to imaginary contributions to the effective potential that are not associated with a physical instability, and therefore spurious. Second, in the limit that $G$ approaches zero, the effective potential minimization condition is logarithmically divergent already at two-loop order, and has increasingly severe power-law singularities at higher loop orders. I resolve both issues by resumming the Goldstone boson contributions to the effective potential. For the resulting resummed effective potential, the minimum value and the minimization condition that gives the vacuum expectation value are obtained in forms that do not involve $G$ at all.
\end{abstract}

\section{Contents}

I. Introduction

II. Effective potential contributions for small $G$

III. Resummation of leading Goldstone contributions

IV. Minimization condition for the effective potential

V. Numerical impact

VI. Outlook 


\section{INTRODUCTION}

A resonance with mass about $126 \mathrm{GeV}$ and properties expected of a minimal Standard Model Higgs scalar boson has been discovered [1-4] at the Large Hadron Collider. One of the theoretical tools useful for understanding the electroweak symmetry breaking dynamics of the minimal Standard Model and its extensions is the effective potential [5-7], which can be used to relate the Higgs field vacuum expectation value (VEV) to the fundamental Lagrangian parameters, and to observable quantities such as the masses of the Higgs bosons, the top quark, and the masses and interactions of the $W$ and $Z$ bosons. On general grounds, the Standard Model Lagrangian parameter should be obtained as accurately as possible. It may be possible to discern the difference between the minimal Higgs Standard Model and more complicated theories, and to gain hints about the mass scale of new physics, and the stability of the Standard Model vacuum state. An interesting feature of the Higgs mass is that the potential is close to a metastable region associated with a very small Higgs selfinteraction coupling at very large energy scales. Some studies of the stability condition that were made before the Higgs discovery are refs. [7-18], and some of the analyses following the Higgs discovery are refs. [19-23].

To fix notation, write the complex doublet Higgs field as

$$
\Phi(x)=\left(\begin{array}{c}
\frac{1}{\sqrt{2}}\left[\phi+H(x)+i G^{0}(x)\right] \\
G^{+}(x)
\end{array}\right) .
$$

Here $\phi$ is the real background field, about which are expanded the real Higgs quantum field $H$, and the real neutral and complex charged Goldstone boson fields $G^{0}$ and $G^{+}=G^{-*}$. The Lagrangian for the Higgs kinetic term and its self-interactions are given by

$$
\mathcal{L}=-\partial^{\mu} \Phi^{\dagger} \partial_{\mu} \Phi-\Lambda-m^{2} \Phi^{\dagger} \Phi-\lambda\left(\Phi^{\dagger} \Phi\right)^{2}
$$

where $m^{2}$ is the Higgs squared mass parameter, and $\lambda$ is the Higgs self-coupling in the normalization to be used in this paper, and the metric signature is $(-+++)$. The fieldindependent vacuum energy density $\Lambda$ is necessary for renormalization scale invariance of the effective potential and a proper treatment of renormalization group improvement [10, 24 28]. The Lagrangian also includes a top-quark Yukawa coupling $y_{t}$, and $S U(3)_{c} \times S U(2)_{L} \times U(1)_{Y}$ gauge couplings $g_{3}, g$, and $g^{\prime}$. The other Yukawa couplings are very small, and can make only a very minor difference even at 1-loop order, and so are neglected. All of the Lagrangian parameters as well as the background field $\phi$ depend on the $\overline{\mathrm{MS}}$ renormalization scale $Q$, 
and logarithms of dimensional quantities are written below in terms of

$$
\overline{\ln }(x) \equiv \ln \left(x / Q^{2}\right)
$$

The effective potential can be evaluated in perturbation theory and written as:

$$
V_{\text {eff }}(\phi)=\sum_{\ell=0}^{\infty} \frac{1}{\left(16 \pi^{2}\right)^{\ell}} V_{\ell}
$$

In this paper, the power of $1 / 16 \pi^{2}$ is used as a signifier for the loop order $\ell$. The tree-level potential is given by:

$$
V_{0}=\Lambda+\frac{m^{2}}{2} \phi^{2}+\frac{\lambda}{4} \phi^{4}
$$

The radiative corrections for $\ell \geq 1$ are obtained from the sum of 1-particle-irreducible vacuum graphs. The Landau gauge is most often used for effective potential calculations, because of the simplifications that the gauge-fixing parameter is not renormalized and there is no mixing between the longitudinal vector modes and the Goldstone modes. In Landau gauge and the $\overline{\mathrm{MS}}$ renormalization scheme based on dimensional regularization, the 1-loop order contribution to the Standard Model effective potential is:

$$
V_{1}=3 f(G)+f(H)-12 f(t)+6 f(W)+W^{2}+3 f(Z)+\frac{1}{2} Z^{2},
$$

where

$$
f(x) \equiv \frac{x^{2}}{4}[\overline{\ln }(x)-3 / 2]
$$

and the field-dependent running squared masses are

$$
\begin{aligned}
G & =m_{G^{0}}^{2}=m_{G^{ \pm}}^{2}=m^{2}+\lambda \phi^{2}, \\
H & =m_{H}^{2}=m^{2}+3 \lambda \phi^{2}, \\
t & =m_{t}^{2}=y_{t}^{2} \phi^{2} / 2, \\
W & =m_{W}^{2}=g^{2} \phi^{2} / 4 \\
Z & =m_{Z}^{2}=\left(g^{2}+g^{\prime 2}\right) \phi^{2} / 4 .
\end{aligned}
$$

The contributions $W^{2}+\frac{1}{2} Z^{2}$ in eq. (1.6) are due to the fact that in dimensional regularization 
the vector fields have $4-2 \epsilon$ components rather than 4 .

The full two-loop order contribution $V_{2}$ in Landau gauge was worked out by Ford, Jack and Jones in [29] for the Standard Model, and for more general theories (including softly-broken supersymmetric models, where regularization by dimensional reduction is used instead of dimensional regularization) in [30]. The 3-loop contribution $V_{3}$ for the Standard Model was obtained in [31] in the approximation that the strong and top Yukawa couplings are much larger than the electroweak couplings and other Yukawa couplings. For completeness, these results are compiled in an Appendix of the present paper in a notation compatible with the discussion below.

Results for more general gauge-fixing conditions are apparently only available at 1-loop order at present. The effective potential itself is gauge-fixing dependent, but physical observables derived from it are not. For discussions of the gauge-fixing dependence of the effective potential from various points of view see refs. [32 47].

The purpose of this paper is to resolve two issues of principle regarding contributions to the effective potential involving the Goldstone bosons. Note that the condition $G=0$ marks the minimum of the tree-level potential $V_{0}$ in eq. (1.5). However, in general $G$ will be non-zero at the minimum of the full effective potential.

The first problem of principle is that there is no reason why $G$ cannot be negative at the minimum of $V_{\text {eff }}$, depending on the choice of renormalization scale $Q$. Indeed, in the case of the Standard Model, $G$ is negative for the perfectly reasonable range $Q \gtrsim 100 \mathrm{GeV}$. This is illustrated in Figure 1.1 for a typical numerical choice of the parameters (in this case taken from ref. [23]):

$$
\begin{aligned}
\lambda\left(M_{t}\right) & =0.12710 \\
y_{t}\left(M_{t}\right) & =0.93697 \\
g_{3}\left(M_{t}\right) & =1.1666 \\
m^{2}\left(M_{t}\right) & =-(93.36 \mathrm{GeV})^{2}, \\
g\left(M_{t}\right) & =0.6483 \\
g^{\prime}\left(M_{t}\right) & =0.3587 .
\end{aligned}
$$

where $Q=M_{t}=173.35 \mathrm{GeV}$ is the input scale. The problem is that the $\overline{\ln }(G)$ terms give rise to an imaginary part of $V_{\text {eff }}$. In general, a complex value of $V_{\text {eff }}$ at its minimum reflects an instability [48], but there is no physical instability here. In practice, this unphysical imaginary part of $V_{\text {eff }}$ has simply been ignored, and the real part is minimized. In principle, this imaginary part is spurious, and a way of making this plain is desirable.

The second problem of principle is that when calculated to fixed loop order $\ell$, the effective 


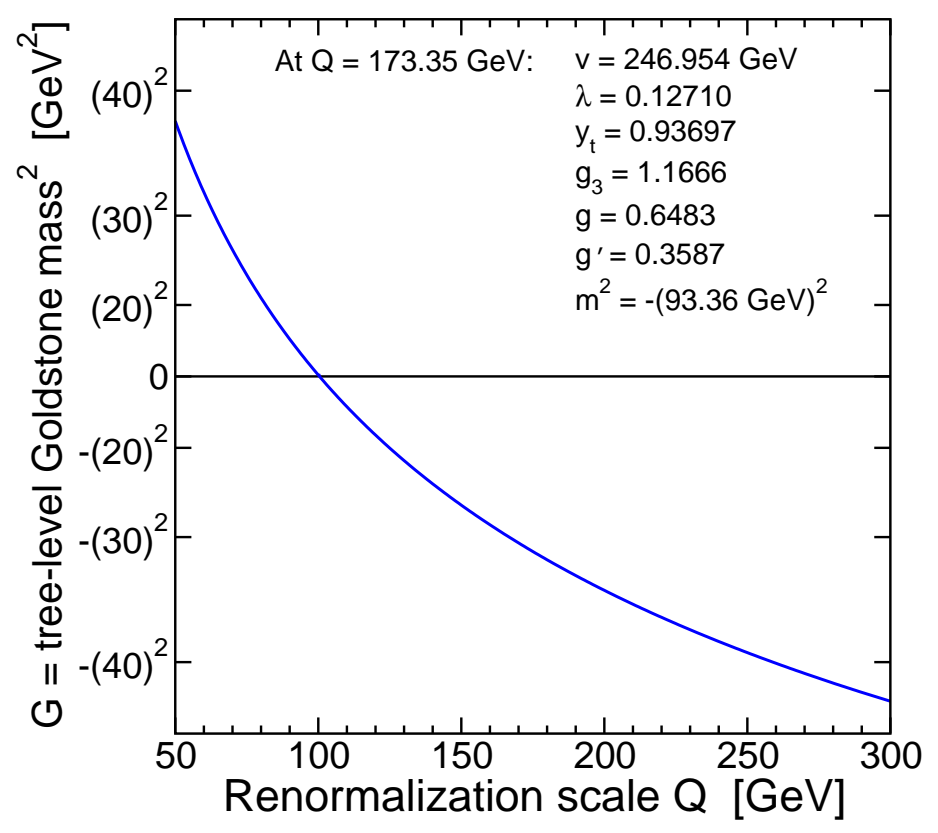

FIG. 1.1: The running of the Landau gauge Standard Model Goldstone boson squared mass $G$, evaluated at the minimum of the effective potential, as a function of the renormalization scale $Q$, for the choice of input parameters specified in the text.

potential diverges for $G \rightarrow 0$. For $\ell=1,2,3$, one finds [31] that $V_{\ell} \sim G^{3-\ell} \overline{\ln } G$, while for $\ell \geq 4$ one has $V_{\ell} \sim G^{3-\ell}$. (The precise results for the leading behavior as $G \rightarrow 0$ will be obtained in the next section.) Thus the effective potential itself has a logarithmic singularity at 3-loop order, with increasingly severe power-law singularities for higher loop orders. The first derivative of the effective potential, which is used in the minimization condition that governs the vacuum expectation value of the Higgs field, therefore has a logarithmic singularity at 2-loop order, and power law singularities for higher loop orders. As we will see below, the numerical impact of these singularities at the known loop order, $\ell=3$, is quite small unless one carefully tunes $G \approx 0$ by the choice of renormalization scale. However, this is still disturbing as a matter of principle. Indeed, one might have expected that a choice of $Q$ that makes $G$ very small would be a particularly good choice, because the pole mass of the Landau gauge Goldstone bosons is 0 , and so $G=0$ corresponds to choosing a renormalization scale such that the radiative corrections to it vanish. However, if one uses the usual perturbative effective potential truncated at any loop order beyond 1-loop order, this is the one renormalization scale choice that one must not make.

The purpose of this paper is to show how the above two problems of principle are eliminated by doing a resummation to all loop orders of the Goldstone contributions that are leading as $G \rightarrow 0$.

\section{EFFECTIVE POTENTIAL CONTRIBUTIONS FOR SMALL $G$}

For the known contributions to the effective potential, the leading behavior as $G \rightarrow 0$ can be isolated as follows. First, at one loop order, we have immediately from eq. (1.6) that 

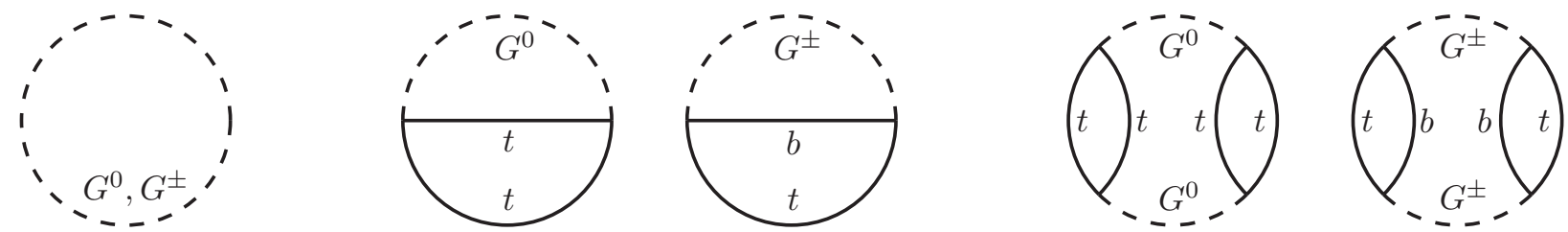

FIG. 2.1: These Feynman diagrams give the leading non-zero contribution to $V_{\text {eff }}$ as $G \rightarrow 0$, at the leading order in $y_{t}$, for 1-loop, 2-loop, and 3-loop orders.

the Goldstone boson contributions are:

$$
V_{1}=3 f(G)+\ldots=\frac{3}{4} G^{2}[\overline{\ln }(G)-3 / 2]+\ldots
$$

In eq. (2.1), the ellipses represent terms with no $G$ dependence. At 2-loop order, using the expansions of the 2-loop integral function $I(x, y, z)$ for small $G$ given in eqs. (A.26)-(A.29) of the Appendix (from eqs. (2.29)-(2.31) of ref. [30]), one finds that

$$
V_{2}=\frac{3}{2} \Delta_{1} G \overline{\ln }(G)+\ldots
$$

where

$$
\Delta_{1}=-6 y_{t}^{2} A(t)+3 \lambda A(H)+\frac{g^{2}}{2}[3 A(W)+2 W]+\frac{g^{2}+g^{\prime 2}}{4}[3 A(Z)+2 Z]
$$

and the 1-loop integral function is defined by

$$
A(x) \equiv x(\overline{\ln } x-1)
$$

(In ref. [30], $A(x)$ was called $J(x)$.) In eq. (2.2), the ellipses represent terms independent of $G$, terms of order $G^{2}$, as well as those proportional to $G$ with no $\overline{\ln }(G)$. Reading directly from eq. (4.38) of ref. [31], one obtains the leading behavior as $G \rightarrow 0$ at 3-loop order:

$$
V_{3}=27 y_{t}^{4} A(t)^{2} \overline{\ln }(G)+\ldots
$$

As noted in ref. [31], the contributions above (that involve $y_{t}$, in the 2-loop case) come from the diagrams shown in Figure 2.1. At higher loop orders, the leading contribution as $G \rightarrow 0$ and at leading order in $y_{t}$ comes from $\ell$-loop order vacuum diagrams consisting of a ring of $\ell-1$ Goldstone boson propagators, interspersed with $\ell-1$ top (for $G^{0}$ ) or top/bottom (for $G^{ \pm}$) 1-loop subdiagrams, as shown in Figure 2.2.

More generally still, the 1-loop subdiagrams can be any 1-particle irreducible sub- 

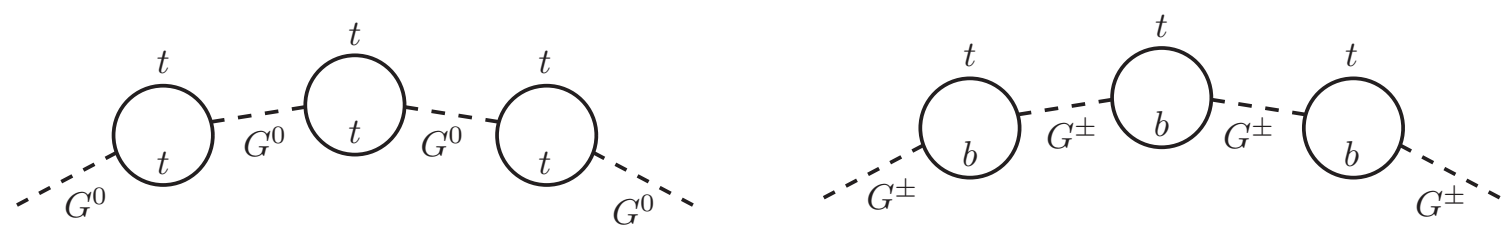

FIG. 2.2: Chains of Goldstone boson propagators interspersed with top and top/bottom loops. Rings of these (and similar diagrams involving loops with $Z, W$, and $H$ ) give rise to the most singular contributions as $G \rightarrow 0$, at any given loop order.

diagrams that involve a squared mass scale that can be treated as parametrically large compared to $G$. In the Standard Model, these includes 1-loop subdiagrams containing $Z$, $W$, and $H$ bosons, as well as multi-loop subdiagrams.

To obtain the leading behavior as $G \rightarrow 0$, one considers the contributions from these diagrams from integrating momenta $p^{\mu}$ flowing around the large rings with $p^{2}$ small compared to the squared mass scale set by the 1-particle-irreducible sub-diagrams. Then the 1-particle-irreducible sub-diagram contributions can be treated as just constant squaredmass insertions in the Goldstone boson propagators, reducing the calculation to a 1-loop integration. The sum of the resulting leading $G \rightarrow 0$ contributions to $V_{\text {eff }}$ at each loop order, including the $\overline{\mathrm{MS}}$ counterterms, is:

$$
V_{\mathrm{eff}}=\frac{3}{16 \pi^{2}} \sum_{n=0}^{\infty} \frac{\Delta^{n}}{n !}\left(\frac{d}{d G}\right)^{n} f(G)+\ldots
$$

where $n=\ell-1$ where $\ell$ is the loop order, and

$$
\Delta=\frac{1}{16 \pi^{2}} \Delta_{1}+\frac{1}{\left(16 \pi^{2}\right)^{2}} \Delta_{2}+\frac{1}{\left(16 \pi^{2}\right)^{3}} \Delta_{3}+\ldots
$$

Equation (2.6) generalizes eqs. (2.1), (2.2), and (2.5). For the purposes of making this particular comparison, $\Delta_{2}$ can be dropped, because ref. [31] retained only the leading order in $y_{t}$ at 3 loops. For the same reason, all but the $y_{t}^{2}$ term in $\Delta_{1}$ can be dropped in comparing the 3-loop order contributions. However, in the future, if more terms are calculated in $V_{\text {eff }}$ at 3-loop order and beyond, then those contributions would become pertinent, as would contributions from other diagrams.

The origin of the prefactor 3 in eq. (2.6) is a factor of 2 for the $G^{ \pm}$rings, and a factor 1 for the $G^{0}$ ring. Despite the fact that the 1-particle-irreducible subdiagrams are different for these two classes of diagrams (e.g. involving top/bottom loops for $G^{ \pm}$, and top loops for $\left.G^{0}\right)$, the quantity $\Delta$ is the same in both cases.

Note that $f^{\prime}(G)=\frac{1}{2} A(G)=\frac{1}{2} G[\overline{\ln }(G)-1]$, and $f^{\prime \prime}(G)=\frac{1}{2} \overline{\ln }(G)$, and the $n$th derivative is $f^{(n)}(G)=\frac{1}{2}(-1)^{n-1}(n-3) ! G^{2-n}$ for $n \geq 3$. Therefore, the leading singular behavior as 
$G \rightarrow 0$ is

$$
\begin{aligned}
& V_{3}=\frac{3}{4}\left(\Delta_{1}\right)^{2} \overline{\ln }(G) \\
& V_{\ell}=-\frac{3\left(-\Delta_{1}\right)^{\ell-1}}{2(\ell-1)(\ell-2)(\ell-3) G^{\ell-3}} \quad(\text { for } \ell>3)
\end{aligned}
$$

\section{RESUMMATION OF LEADING GOLDSTONE CONTRIBUTIONS}

The contributions to $V_{\text {eff }}$ in eq. (2.6) from all loop orders $\ell=n+1$ resum to

$$
V_{\text {eff }}=\frac{3}{16 \pi^{2}} f(G+\Delta)+\ldots
$$

Thus, the result of summing all orders in perturbation theory yields a result which is wellbehaved for all $G$, unlike the result obtained if it is truncated at any finite order in perturbation theory. In fact, at the minimum of the full effective potential, $G+\Delta=0$, and the result of the resummation of this class of terms vanishes. Therefore, if $V_{\text {eff }}$ has been evaluated at some finite $\ell$-loop order in perturbation theory, a sensible result can be obtained by simply subtracting off the first $\ell$ terms in the series eq. (2.6), and then adding back in the resummed version of the same series, eq. (3.1). If the effective potential $V_{\text {eff }}$ has been calculated to loop order $\ell$, then the resummed effective potential is

$$
\widehat{V}_{\mathrm{eff}}=V_{\mathrm{eff}}+\frac{3}{16 \pi^{2}}\left[f(G+\Delta)-\sum_{n=0}^{\ell-1} \frac{\Delta^{n}}{n !}\left(\frac{d}{d G}\right)^{n} f(G)\right]
$$

The result is free of the offending leading singular contributions as $G \rightarrow 0$.

Further resummation may be necessary to account for other terms that are singular as $G \rightarrow 0$ but sub-leading at a given loop order, but these do not arise in the effective potential approximation that has been calculated so far. If we use $V_{\text {eff }}$ to refer to the usual full 2-loop and leading 3-loop Standard Model effective potential as computed in refs. [29] and [31], then the appropriate resummed version from eq. (3.2) is:

$$
\widehat{V}_{\text {eff }}=V_{\text {eff }}+\frac{3}{16 \pi^{2}}[f(G+\Delta)-f(G)]-\frac{1}{\left(16 \pi^{2}\right)^{2}} \frac{3 \Delta_{1}}{2} A(G)-\frac{1}{\left(16 \pi^{2}\right)^{3}} 27 y_{t}^{4} A(t)^{2} \overline{\ln }(G)
$$

Here I have taken $\left(\Delta_{1}\right)^{2}=36 y_{+}^{4} A(t)^{2}$ in the 3-loop part, because only the leading order in

$y_{t}$ for $V_{\text {eff }}$ was included in ref. [31]. For the same reason, the 3-loop order term involving $\Delta_{2}$ is dropped here. The effect of the 1-loop correction in eq. (3.3) is to replace the tree-level 
field-dependent Goldstone boson squared mass by its pole squared mass, which vanishes at the minimum of the full effective potential in Landau gauge. The 3-loop order term simply cancels the corresponding $\overline{\ln }(G)$ contribution found in ref. [31]. I propose that the resummed version of the effective potential, $\widehat{V}_{\text {eff }}$, should be used instead of the usual $V_{\text {eff }}$.

\section{MINIMIZATION CONDITION FOR THE EFFECTIVE POTENTIAL}

For the usual effective potential $V_{\text {eff }}$, the minimization condition that relates the vacuum expectation value $v=\phi_{\min }$ to the Lagrangian parameters is

$$
G=m^{2}+\lambda v^{2}=-\frac{1}{16 \pi^{2}} \delta_{1}-\frac{1}{\left(16 \pi^{2}\right)^{2}} \delta_{2}-\frac{1}{\left(16 \pi^{2}\right)^{3}} \delta_{3}-\ldots,
$$

where the correction at $\ell$-loop order is:

$$
\delta_{\ell}=\left.\frac{1}{\phi} \frac{\partial}{\partial \phi} V_{\ell}\right|_{\phi=v}
$$

(In the remainder of this section, I will use $\phi=v$, because all equations hold only at the minimum of the potential.) Explicitly, at 1-loop order, one has from eq. (1.6):

$$
\delta_{1}=3 \lambda A(G)-6 y_{t}^{2} A(t)+3 \lambda A(H)+\frac{g^{2}}{2}[3 A(W)+2 W]+\frac{g^{2}+g^{\prime 2}}{4}[3 A(Z)+2 Z],
$$

and the higher loop order contributions can be obtained by taking derivatives of the results of ref. [29] at 2-loop order, and from ref. [31] at 3-loop order for terms that are leading order in $g_{3}$ and $y_{t}$. Note that $\delta_{1}$ differs from the quantity $\Delta_{1}$, given in eq. (2.3) above, only by the inclusion of the first term, $3 \lambda A(G)$. At higher loop orders $\ell \geq 2$, it is useful to note the leading dependence on $G$ as $G \rightarrow 0$ :

$$
\begin{aligned}
& \delta_{2}=3 \lambda \Delta_{1} \overline{\ln }(G)-9 y_{t}^{4} \overline{\ln }(t) A(G)+\ldots \\
& \delta_{3}=54 y_{t}^{4}\left[\frac{\lambda A(t)^{2}}{G}+y_{t}^{2} A(t) \overline{\ln }(t) \overline{\ln }(G)\right]+\ldots
\end{aligned}
$$

Equation (4.4) can be obtained using eqs. (A.1)-(A.21) and (A.26)-(A.29) in the Appendix, and eq. (4.5) can be obtained from eq. (A.30). In $\delta_{2}$, the ellipses includes terms that do not depend on $G$, terms with a linear factor of $G$ but suppressed by $g, g^{\prime}, \lambda$ or not containing $\overline{\ln }(G)$, and terms quadratic or higher order in $G$. In $\delta_{3}$, the ellipses represents terms that are finite as $G \rightarrow 0$ or suppressed by $g, g^{\prime}, \lambda$. 
The condition for minimization of $\widehat{V}_{\text {eff }}$ defined in eq. (3.3) is:

$$
G=m^{2}+\lambda v^{2}=-\frac{1}{16 \pi^{2}} \Delta_{1}-\frac{1}{\left(16 \pi^{2}\right)^{2}} \Delta_{2}-\frac{1}{\left(16 \pi^{2}\right)^{3}} \Delta_{3}-\ldots
$$

where

$$
\Delta_{\ell}=\frac{1}{v} \frac{\partial}{\partial v} \widehat{V}_{\ell}
$$

with $\widehat{V}_{\text {eff }}=\sum_{\ell} \frac{1}{\left(16 \pi^{2}\right)^{\ell}} \widehat{V}_{\ell}$. Consider the difference between the minimization conditions for $V_{\text {eff }}$ and $\widehat{V}_{\text {eff }}$. First, note that the term proportional to $f(G+\Delta)$ gives no contribution, because $f^{\prime}(G+\Delta)=\frac{1}{2}(G+\Delta)(\overline{\ln }(G+\Delta)-1)$ vanishes at the minimum of the potential, where $G+\Delta=0$. One therefore finds from eq. (3.3):

$$
\begin{aligned}
\frac{1}{v} \frac{\partial}{\partial v}\left(\widehat{V}_{\text {eff }}-V_{\text {eff }}\right)= & -\frac{1}{16 \pi^{2}} 3 \lambda A(G)-\frac{1}{\left(16 \pi^{2}\right)^{2}}\left[3 \lambda \Delta_{1} \overline{\ln }(G)+\frac{3}{2} A(G) \frac{1}{v} \frac{\partial \Delta_{1}}{\partial v}\right] \\
& -\frac{1}{\left(16 \pi^{2}\right)^{3}} 54 y_{t}^{4}\left[\frac{\lambda A(t)^{2}}{G}+y_{t}^{2} A(t) \overline{\ln }(t) \overline{\ln }(G)\right] .
\end{aligned}
$$

Note that the apparently 2-loop term containing $A(G)$ is actually of 3-loop order, because $A(G)$ contains a factor of $G$, which at the minimum of the potential is equal to $-\frac{1}{16 \pi^{2}} \Delta_{1}+\ldots$ Therefore, in eq. (4.8), to be consistent we should keep only the part of $\frac{1}{v} \frac{\partial \Delta_{1}}{\partial v}$ that involves the top Yukawa coupling:

$$
\frac{1}{v} \frac{\partial \Delta_{1}}{\partial v}=-6 y_{t}^{4} \overline{\ln }(t)+\ldots
$$

So, one obtains from eq. (4.8):

$$
\begin{aligned}
& \Delta_{1}=\delta_{1}-3 \lambda A(G) \\
& \Delta_{2}=\delta_{2}-3 \lambda \Delta_{1} \overline{\ln }(G)+9 y_{t}^{4} \overline{\ln }(t) A(G), \\
& \Delta_{3}=\delta_{3}-54 y_{t}^{4}\left[\frac{\lambda A(t)^{2}}{G}+y_{t}^{2} A(t) \overline{\ln }(t) \overline{\ln }(G)\right] .
\end{aligned}
$$

This shows that the $G$-dependent terms of eqs. (4.3)-(4.5) neatly cancel, up to the order that has been calculated.

The resulting $\Delta_{1}$ does not explicitly depend on $G$, but depends on $m^{2}$ through $H$. A 
further refinement of the minimization condition can be made by writing

$$
H=h+G
$$

where, from eq. (1.9),

$$
h=2 \lambda v^{2}
$$

at the minimum of the potential, and then iteratively replacing the $G$ dependence using

$$
G^{n} \overline{\ln }^{p}(G)=\left(-\frac{1}{16 \pi^{2}} \Delta_{1}-\frac{1}{\left(16 \pi^{2}\right)^{2}} \Delta_{2}-\ldots\right)^{n} \overline{\ln }^{p}(G)
$$

in $\Delta_{1}, \Delta_{2}$, and $\Delta_{3}$. (Note that logarithms of $G$ are left alone, to cancel amongst themselves.) In doing so, one consistently drops terms of 4-loop order as well as terms of 3-loop order that are suppressed by $g, g^{\prime}$ or $\lambda$. Thus, for example, the 1-loop contribution involving $H$ is rewritten using

$$
\begin{aligned}
A(H) & =A(h)+G A^{\prime}(h)+\frac{G^{2}}{2} A^{\prime \prime}(h)+\ldots \\
& =A(h)-\frac{1}{16 \pi^{2}} \Delta_{1} \overline{\ln }(h)+\frac{1}{\left(16 \pi^{2}\right)^{2}}\left[\frac{\left(\Delta_{1}\right)^{2}}{2 h}-\Delta_{2} \overline{\ln }(h)\right]+\ldots
\end{aligned}
$$

Because this is multiplied by $3 \lambda / 16 \pi^{2}$ in the minimization condition, the $\Delta_{2}$ term should now be dropped, but the $\left(\Delta_{1}\right)^{2} / 2 h$ term is partially kept, because $3 \lambda\left(\Delta_{1}\right)^{2} / 2 h=27 y_{t}^{4} A(t)^{2} / v^{2}+$ ... The self-consistent elimination of $G$ from the right side of the minimization condition shifts contributions that were originally proportional to $G^{n}$ up in loop order by $n$, where they can often therefore be dismissed. (Note that this iterative elimination of $G$ from the right-hand side of the minimization condition would not have been possible without first eliminating by resummation the terms that behave like $\overline{\ln }(G)$ and $1 / G$ as $G \rightarrow 0$.)

Following the procedure described above, I find as the condition for minimization of $\widehat{V}_{\text {eff }}$ :

$$
G=m^{2}+\lambda v^{2}=-\frac{1}{16 \pi^{2}} \widehat{\Delta}_{1}-\frac{1}{\left(16 \pi^{2}\right)^{2}} \widehat{\Delta}_{2}-\frac{1}{\left(16 \pi^{2}\right)^{3}} \widehat{\Delta}_{3}-\ldots
$$

where the $\widehat{\Delta}_{\ell}$ depend on the VEV $v$ and the couplings $\lambda, y_{t}, g_{3}, g, g^{\prime}$, or on $h, t, W, Z$, but do not depend at all on $G$ or $m^{2}$. The results are:

$$
\widehat{\Delta}_{1}=-6 y_{t}^{2} A(t)+3 \lambda A(h)+\frac{g^{2}}{2}[3 A(W)+2 W]+\frac{g^{2}+g^{\prime 2}}{4}[3 A(Z)+2 Z],
$$




$$
\begin{aligned}
& \widehat{\Delta}_{2}=\frac{\left(3 g^{2}-g^{\prime 2}\right)\left(33 g^{4}+22 g^{2} g^{\prime 2}+g^{\prime 4}\right)}{8\left(g^{2}+g^{\prime 2}\right)} I(W, W, Z) \\
& +\left[\frac{\left(9 g^{4}+66 g^{2} g^{\prime 2}-7 g^{\prime 4}\right) y_{t}^{2}}{6\left(g^{2}+g^{\prime 2}\right)}-\frac{3}{4} g^{4}+\frac{1}{2} g^{2} g^{\prime 2}-\frac{17}{12} g^{\prime 4}\right] I(t, t, Z) \\
& +\left[\frac{3\left(g^{2}+g^{\prime 2}\right)^{3}}{32\left(g^{2}+g^{\prime 2}-2 \lambda\right)}-\frac{15}{16}\left(g^{2}+g^{\prime 2}\right)^{2}+\frac{11}{4} \lambda\left(g^{2}+g^{\prime 2}\right)-7 \lambda^{2}\right] I(h, Z, Z) \\
& +\left[\frac{3 g^{6}}{16\left(g^{2}-2 \lambda\right)}-\frac{15}{8} g^{4}+\frac{11}{2} \lambda g^{2}-14 \lambda^{2}\right] I(h, W, W) \\
& +y_{t}^{2}\left[\frac{27}{2} y_{t}^{2}-18 \lambda\right] I(h, t, t)-12 \lambda y_{t}^{2} I(0,0, t)-15 \lambda^{2} I(h, h, h) \\
& -6 \lambda^{2} I(0,0, h)+\frac{3}{4} \lambda\left(g^{2}+g^{\prime 2}+8 \lambda\right) I(0, h, Z)+\frac{3}{2} \lambda\left(g^{2}+8 \lambda\right) I(0, h, W) \\
& +\frac{3 \lambda\left(2 g^{2}+g^{\prime 2}\right)^{3}}{2\left(g^{2}+g^{\prime 2}\right)^{2}} I(0, W, Z)+\left(6 y_{t}^{4}+3 g^{2} y_{t}^{2}-3 g^{4}\right) I(0, t, W) \\
& +\left[\frac{15}{2} \lambda g^{2}-9 g^{4}-\frac{3 \lambda g^{4}\left(3 g^{2}+2 g^{\prime 2}\right)}{2\left(g^{2}+g^{\prime 2}\right)^{2}}\right] I(0,0, W) \\
& +\left[\frac{9 \lambda g^{4}}{2\left(g^{2}+g^{\prime 2}\right)}-3 g^{2} \lambda-\frac{21}{4} g^{4}-\frac{g^{2} g^{\prime 2}}{2}-\frac{103}{12} g^{\prime 4}\right] I(0,0, Z) \\
& +\left[\frac{7}{2} \lambda-\frac{g^{2}+g^{\prime 2}}{2}+\frac{3\left(g^{2}+g^{\prime 2}\right)^{2}}{8\left(2 \lambda-g^{2}-g^{\prime 2}\right)}\right] A(Z)^{2} / v^{2} \\
& +\left[7 \lambda-\frac{35}{2} g^{2}+\frac{g^{\prime 2}}{2}+\frac{24 g^{4}}{g^{2}+g^{\prime 2}}+\frac{3 g^{4}}{4\left(2 \lambda-g^{2}\right)}\right] A(W)^{2} / v^{2} \\
& +\left[\frac{6 \lambda\left(8 g^{4}+8 g^{2} g^{\prime 2}+g^{\prime 4}\right)}{\left(g^{2}+g^{\prime 2}\right)^{2}}+\frac{15 g^{4}-10 g^{2} g^{\prime 2}-g^{\prime 4}}{g^{2}+g^{\prime 2}}\right] A(W) A(Z) / v^{2} \\
& -\frac{2\left(9 g^{4}-6 g^{2} g^{\prime 2}+17 g^{\prime 4}\right)}{3\left(g^{2}+g^{\prime 2}\right)} A(t) A(Z) / v^{2}+12\left(y_{t}^{2}-g^{2}\right) A(t) A(W) / v^{2} \\
& +\left[96 g_{3}^{2}+15 y_{t}^{2}+6 \lambda+\frac{9 g^{4}+90 g^{2} g^{\prime 2}+17 g^{\prime 4}}{3\left(g^{2}+g^{\prime 2}\right)}\right] A(t)^{2} / v^{2} \\
& +\left[\frac{3}{4}\left(g^{2}+g^{\prime 2}\right)-4 \lambda+\frac{3\left(g^{2}+g^{\prime 2}\right)^{2}}{8\left(g^{2}+g^{\prime 2}-2 \lambda\right)}\right] A(h) A(Z) / v^{2} \\
& +\left[\frac{3}{2} g^{2}-8 \lambda+\frac{3 g^{4}}{4\left(g^{2}-2 \lambda\right)}\right] A(h) A(W) / v^{2}-9 y_{t}^{2} A(h) A(t) / v^{2} \\
& +\left[\frac{3\left(g^{2}+g^{\prime 2}\right)^{3}}{16\left(2 \lambda-g^{2}-g^{\prime 2}\right)}+\frac{\lambda\left(g^{\prime 2}-23 g^{2}\right)}{2}-\frac{y_{t}^{2}\left(63 g^{4}+30 g^{2} g^{\prime 2}+95 g^{\prime 4}\right)}{12\left(g^{2}+g^{\prime 2}\right)}\right. \\
& \left.+\frac{6 g^{6}}{g^{2}+g^{\prime 2}}+\frac{29}{48} g^{4}+4 g^{2} g^{\prime 2}+\frac{455}{48} g^{\prime 4}\right] A(Z) \\
& +g^{2}\left[\frac{3 g^{4}}{8\left(2 \lambda-g^{2}\right)}-\frac{21}{2} y_{t}^{2}-\frac{3 g^{2}\left(\lambda+4 g^{2}\right)}{g^{2}+g^{2}}+\frac{605}{24} g^{2}+\frac{13}{8} g^{\prime 2}-8 \lambda\right] A(W) \\
& +y_{t}^{2}\left[16 g_{3}^{2}-12 y_{t}^{2}+24 \lambda-\frac{91}{3} g^{2}+\frac{11}{3} g^{\prime 2}+\frac{64 g^{4}}{3\left(g^{2}+g^{\prime 2}\right)}\right] A(t)
\end{aligned}
$$




$$
\begin{aligned}
+ & {\left[\frac{3\left(g^{2}+g^{\prime 2}\right)^{3}}{32\left(2 \lambda-g^{2}-g^{\prime 2}\right)}+\frac{3 g^{6}}{16\left(2 \lambda-g^{2}\right)}-\frac{21}{2} y_{t}^{4}+9 \lambda y_{t}^{2}+24 \lambda^{2}\right.} \\
& \left.-\frac{7}{4} \lambda\left(3 g^{2}+g^{\prime 2}\right)+\frac{9}{2} g^{4}+3 g^{2} g^{\prime 2}+\frac{3}{2} g^{\prime 4}\right] A(h) \\
+ & {\left[24 g_{3}^{2} y_{t}^{4}+9 y_{t}^{6}+6 y_{t}^{4} \lambda+\frac{y_{t}^{4}\left(18 g^{4}+87 g^{2} g^{\prime 2}+5 g^{\prime 4}\right)}{6\left(g^{2}+g^{\prime 2}\right)}+\frac{9 g^{8}}{32\left(g^{2}-2 \lambda\right)}\right.} \\
+ & \frac{9\left(g^{2}+g^{\prime 2}\right)^{4}}{64\left(g^{2}+g^{\prime 2}-2 \lambda\right)}+\frac{3 g^{6}\left(8 g^{2}+\lambda\right)}{8\left(g^{2}+g^{\prime 2}\right)}-60 \lambda^{3}+\lambda^{2}\left(6 g^{2}+2 g^{\prime 2}-18 y_{t}^{2}\right) \\
& -\frac{91}{48} y_{t}^{2} g^{\prime 4}+\frac{23}{8} y_{t}^{2} g^{2} g^{\prime 2}-\frac{27}{16} y_{t}^{2} g^{4}+\frac{93}{16} \lambda g^{4}+\frac{7}{8} \lambda g^{2} g^{\prime 2}+\frac{\lambda g^{\prime 4}}{16} \\
+ & \left.\frac{199}{64} g^{6}-\frac{551}{192} g^{4} g^{\prime 2}-\frac{773}{192} g^{2} g^{\prime 4}-\frac{497}{192} g^{\prime 6}\right] v^{2}, \\
\widehat{\Delta}_{3}= & g_{3}^{4} y_{t}^{4} v^{2}\left[1036.23-974.20 \overline{\ln }(t)+592 \overline{\ln }^{2}(t)-184 \overline{\ln }^{3}(t)\right] \\
+ & g_{3}^{2} y_{t}^{6} v^{2}\left[-169.84+860.93 \overline{\ln }(t)-270 \overline{\ln }^{2}(t)+60 \overline{\ln }^{3}(t)\right] \\
+ & y_{t}^{8} v^{2}\left[-82.91-753.02 \overline{\ln }(t)+36 \overline{\ln }(h) \overline{\ln }^{2}(t)+\frac{657}{8} \overline{\ln }^{2}(t)\right. \\
+ & \left.54 \overline{\ln }(h) \overline{\ln }^{2}(t)-\frac{225}{4} \overline{\ln }^{3}(t)\right]+\ldots
\end{aligned}
$$

Here, the ellipses represent terms suppressed by $g, g^{\prime}$, or $\lambda$. The analytical versions of the decimal coefficients in eq. (4.21) are:

$$
\begin{aligned}
1036.23 \approx & \frac{8170}{9}+48 \zeta(3)+\frac{176}{135} \pi^{4}+\frac{64}{9} \ln ^{2}(2)\left[\pi^{2}-\ln ^{2}(2)\right]-\frac{512}{3} \operatorname{Li}_{4}(1 / 2), \\
-974.20 \approx & 32 \zeta(3)-3038 / 3 \\
-169.84 \approx & -\frac{1172}{3}-\frac{40}{3} \pi^{2}-96 \zeta(3)+\frac{124}{15} \pi^{4}+\frac{128}{3} \ln ^{2}(2)\left[\pi^{2}-\ln ^{2}(2)\right] \\
& -1024 \operatorname{Li}_{4}(1 / 2), \\
860.93 \approx & 454+12 \pi^{2}+240 \zeta(3), \\
-82.91 \approx & \frac{3979}{8}+\frac{37}{8} \pi^{2}-\frac{909}{2} \zeta(3)-\frac{22}{15} \pi^{4}-8 \ln ^{2}(2)\left[\pi^{2}-\ln ^{2}(2)\right]+192 \operatorname{Li}_{4}(1 / 2), \\
-753.02 \approx & -\frac{5145}{8}-\frac{27}{4} \pi^{2}-36 \zeta(3) .
\end{aligned}
$$

Having found the minimization condition in a form that does not depend on $G$, one can now write the value of $\widehat{V}_{\text {eff }}$ at its minimum, again eliminating all $G$ and $m^{2}$ dependence by the same procedure. The result is:

$$
\widehat{V}_{\text {eff,min }}=\sum_{\ell=0}^{\infty} \frac{1}{\left(16 \pi^{2}\right)^{\ell}} \widehat{V}_{\ell, \min },
$$


where

$$
\begin{aligned}
& \widehat{V}_{0, \min }=\Lambda-\lambda v^{4} / 4, \\
& \widehat{V}_{1, \min }=3 t^{2}[\overline{\ln }(t)-1 / 2]-\frac{1}{2} h^{2}[\overline{\ln }(h)-3 / 4]-\frac{3}{2} W^{2}[\overline{\ln }(W)+1 / 6] \\
& -\frac{3}{4} Z^{2}[\overline{\ln }(Z)+1 / 6] \\
& \widehat{V}_{2, \min }=-3 \lambda^{2} v^{2}[I(h, h, h)+I(h, 0,0)]-\frac{3}{4} \lambda A(h)^{2} \\
& +3 y_{t}^{2}\left[(2 t-h / 2) I(h, t, t)+t I(0,0, t)+A(t)^{2}\right] \\
& +\frac{g^{2}+g^{\prime 2}}{8} f_{S S V}(0, h, Z)+\frac{\left(g^{2}-g^{\prime 2}\right)^{2}}{8\left(g^{2}+g^{\prime 2}\right)} f_{S S V}(0,0, Z)+\frac{g^{2}}{4} f_{S S V}(0, h, W) \\
& +\frac{g^{2}}{4} f_{S S V}(0,0, W)+\frac{g^{2} g^{\prime 2} v^{2}}{4\left(g^{2}+g^{\prime 2}\right)}\left[g^{\prime 2} f_{V V S}(W, Z, 0)+g^{2} f_{V V S}(0, W, 0)\right] \\
& +\frac{g^{4} v^{2}}{8} f_{V V S}(W, W, h)+\frac{\left(g^{2}+g^{\prime 2}\right)^{2} v^{2}}{16} f_{V V S}(Z, Z, h) \\
& +V_{F F V}+V_{\text {gauge }}-\frac{v^{2}}{2} \widehat{\Delta}_{2} \\
& \widehat{V}_{3, \text { min }}=g_{3}^{4} t^{2}\left\{184 \overline{\ln }^{3}(t)-316 \overline{\ln }^{2}(t)+\left[\frac{434}{3}-32 \zeta(3)\right] \overline{\ln }(t)+\frac{293}{9}\right. \\
& \left.-64 \zeta(3)-\frac{176}{135} \pi^{4}-\frac{64}{9} \ln ^{2}(2)\left[\pi^{2}-\ln ^{2}(2)\right]+\frac{512}{3} \operatorname{Li}_{4}(1 / 2)\right\} \\
& +g_{3}^{2} y_{t}^{2} t^{2}\left\{-60 \overline{\ln }^{3}(t)+180 \overline{\ln }^{2}(t)+\left[-94-12 \pi^{2}-240 \zeta(3)\right] \overline{\ln }(t)-\frac{49}{3}\right. \\
& \left.+\frac{22}{3} \pi^{2}-24 \zeta(3)-\frac{124}{15} \pi^{4}-\frac{128}{3} \ln ^{2}(2)\left[\pi^{2}-\ln ^{2}(2)\right]+1024 \operatorname{Li}_{4}(1 / 2)\right\} \\
& +y_{t}^{4} t^{2}\left\{\frac{333}{4} \overline{\ln }^{3}(t)+\left[-\frac{405}{4}-72 \overline{\ln }(h)\right] \overline{\ln }^{2}(t)\right. \\
& +\left[\frac{5361}{8}+\frac{39}{4} \pi^{2}+36 \zeta(3)-72 \overline{\ln }(h)\right] \overline{\ln }(t)-\frac{3029}{16}-\frac{11}{4} \pi^{2} \\
& \left.+\frac{22}{15} \pi^{4}+\frac{945}{2} \zeta(3)+8 \ln ^{2}(2)\left[\pi^{2}-\ln ^{2}(2)\right]-192 \operatorname{Li}_{4}(1 / 2)\right\},
\end{aligned}
$$

Here $v, h, t, W, Z$ are understood to be evaluated at the solution of the minimization condition, given by eqs. (4.18)-(4.21). Although eqs. (4.28)-(4.32) have only been computed in Landau gauge here, the value of the effective potential at its minimum is in principle a physical observable and does not depend on the choice of gauge fixing, unlike the VEV itself.

Renormalization group scale invariance provides an important and non-trivial check on the results above. If one acts on each side of eq. (4.18) with

$$
Q \frac{d}{d Q}=Q \frac{\partial}{\partial Q}-\gamma_{\phi} v \frac{\partial}{\partial v}+\sum_{X} \beta_{X} \frac{\partial}{\partial X}
$$


where $X=\left\{\lambda, y_{t}, g_{3}, g, g^{\prime}, m^{2}\right\}$, then the results must match, up to terms of 3-loop order suppressed by $\lambda, g, g^{\prime}$ and terms of 4-loop order. I have checked this, using the beta functions and scalar field anomalous dimension $\gamma_{\phi}$ given at the pertinent orders in eqs. (A.32)-(A.45) in the Appendix. Equations (A.23)-(A.25) are also useful in conducting this check. Similarly, I have checked that acting with eq. (4.33) on eq. (4.28) gives 0 , up to terms of 3-loop order suppressed by $\lambda, g, g^{\prime}$ and terms of 4-loop order, as required. In that check, one has instead $X=\left\{\lambda, y_{t}, g_{3}, g, g^{\prime}, \Lambda\right\}$, with the beta function for the field-independent vacuum energy density given by eqs. (A.46)- (A.47).

To conclude this section, I remark on a different expansion procedure that eliminates the Goldstone boson dependence of the minimization condition for the effective potential, proposed in ref. [45] to avoid spurious gauge dependence in physical quantities in the context of a more general gauge-fixing and finite temperature field theory with applications to baryogenesis. The idea, called the " $\hbar$ expansion" in ref. [45], is to first write an expansion of the VEV in the same way as the effective potential:

$$
v=\phi_{\min }=\phi_{0}+\frac{1}{16 \pi^{2}} \phi_{1}+\frac{1}{\left(16 \pi^{2}\right)^{2}} \phi_{2}+\frac{1}{\left(16 \pi^{2}\right)^{3}} \phi_{3}+\ldots
$$

and then to demand that, after expanding in the loop-counting parameter (here $1 / 16 \pi^{2}$ ), the contributions to the derivative of $V_{\text {eff }}$ vanish separately at each loop order. Then $\phi_{0}$ minimizes the tree-level potential $V_{0}$, so that $m^{2}+\lambda \phi_{0}^{2}=0$ and $V_{0}^{\prime \prime}\left(\phi_{0}\right)=2 \lambda \phi_{0}^{2}$, with the subsequent terms in the expansion of the VEV given by:

$$
\begin{aligned}
& \phi_{1}=-V_{1}^{\prime} / V_{0}^{\prime \prime}, \\
& \phi_{2}=-\left[V_{2}^{\prime}+\phi_{1} V_{1}^{\prime \prime}+\frac{1}{2} \phi_{1}^{2} V_{0}^{\prime \prime \prime}\right] / V_{0}^{\prime \prime}, \\
& \phi_{3}=-\left[V_{3}^{\prime}+\phi_{1} V_{2}^{\prime \prime}+\phi_{2} V_{1}^{\prime \prime}+\frac{1}{2} \phi_{1}^{2} V_{1}^{\prime \prime \prime}+\phi_{1} \phi_{2} V_{0}^{\prime \prime \prime}+\frac{1}{6} \phi_{1}^{3} V_{0}^{\prime \prime \prime \prime}\right] / V_{0}^{\prime \prime},
\end{aligned}
$$

etc., with all of the derivatives of $V_{\ell}$ on the right-hand sides evaluated at the tree-level minimum, $\phi_{0}=\sqrt{-m^{2} / \lambda}$. Each of the individual terms $V_{1}^{\prime \prime}, V_{1}^{\prime \prime \prime}, V_{2}^{\prime}, V_{2}^{\prime \prime}$, and $V_{3}^{\prime}$ diverges as $\phi \rightarrow \phi_{0}$, but one can check that the combination $\phi_{2}$ is well-behaved in this limit, and that $\phi_{3}$ is well-behaved up to the approximation corresponding to the known calculation of $V_{3}$ in ref. [31]. The result is therefore indeed free of spurious imaginary parts and the $G \rightarrow 0$ problems, by construction. However, the result is organized quite differently from that of the present paper, eqs. (4.18)-(4.21); it corresponds to a perturbative solution of the minimization conditions, expanded around the tree-level minimum. It is interesting to look at the explicit results for the expansion method of ref. [45], for simplicity in the limit that 
$y_{t}$ is much larger than $\lambda$, and $g, g^{\prime}=0$ :

$$
\begin{aligned}
\phi_{1} & =\frac{3 y_{t}^{4}}{2 \lambda}\left[\overline{\ln }\left(t_{0}\right)-1\right] \phi_{0}+\ldots, \\
\phi_{2} & =\frac{9 y_{t}^{8}}{8 \lambda^{2}}\left[\overline{\ln }\left(t_{0}\right)-1\right]\left[1+3 \overline{\ln }\left(t_{0}\right)\right] \phi_{0}+\ldots, \\
\phi_{3} & =\frac{27 y_{t}^{12}}{16 \lambda^{3}}\left[\overline{\ln }\left(t_{0}\right)-1\right]\left[-3+6 \overline{\ln }\left(t_{0}\right)+5 \overline{\ln }^{2}\left(t_{0}\right)\right] \phi_{0}+\ldots,
\end{aligned}
$$

with $t_{0}=y_{t}^{2} \phi_{0}^{2} / 2$. The presence of powers of $\lambda$ in the denominators is due to $V_{0}^{\prime \prime}=2 \lambda \phi_{0}^{2}$. This shows that, due to expanding around the tree-level VEV, the expansion parameter is effectively $\frac{y_{t}^{4}}{16 \pi^{2} \lambda}$, rather than the usual perturbative expansion parameters $\frac{y_{t}^{2}}{16 \pi^{2}}$ and $\frac{\lambda}{16 \pi^{2}}$. Correspondingly, in this approach some of the information present in the known $V_{0}$, $V_{1}, V_{2}$, and $V_{3}$ evaluated at the minimum of the full effective potential is postponed to the contributions $\phi_{\ell}$ with $\ell \geq 4$. With the presently known approximation to $V_{3}$ found in ref. [31], the finiteness of $\phi_{3}$ [as the limit $\phi \rightarrow \phi_{0}$ is taken in the derivatives of $V_{\ell}$ in eq. (4.37)] only works up to the order $y_{t}^{8} \phi_{0} / \lambda$ (in an expansion of $\phi_{3}$ in small $\lambda / y_{t}^{2}$ ). A further calculation of subleading corrections to $V_{3}$ in the expansion in $\lambda$ would be necessary to make well-defined the $\phi_{3}$ contributions of order $y_{t}^{6} \phi_{0}$ and $g_{3}^{2} y_{t}^{4} \phi_{0}$. Despite these formal issues, I have checked that in practice the numerical result of applying the expansion procedure of ref. [45] as in eqs. (4.34)-(4.37) above, with all known effective potential contributions included, agrees very well with the expansion found in the present paper, eqs. (4.18)-(4.21). For the input parameters of eqs. (1.13)-(1.18), the two methods agree on the predicted VEV to within 20 $\mathrm{MeV}$.

\section{NUMERICAL IMPACT}

The numerical effect of the resummation is very small for almost all choices of the renormalization scale. This is illustrated in Figure 5.1. In the left panel, the input parameters are specified by eqs. (1.13)-(1.18) at the input scale $Q=173.35 \mathrm{GeV}$. These are then run using the full 3-loop renormalization group equations [50]-[56] to the scale $Q$, where the minimization of the effective potential gives the VEV $v$. Three different approximations are shown: the full 2-loop order $V_{\text {eff }}$ of ref. [29], the same result including partial 3-loop order contributions from [31], and the result after resummation, using eqs. (4.18)-(4.21). (For the first two approximations, the effective potential is complex for all $Q \gtrsim 100.4 \mathrm{GeV}$, so it is the real part that is minimized.) The 3-loop order result without resummation has a severe numerical instability, marked by a failure to converge of the iterative solution for $v$, near the renormalization scale at which $G$ crosses through 0 (compare Figure 1.1). This is 

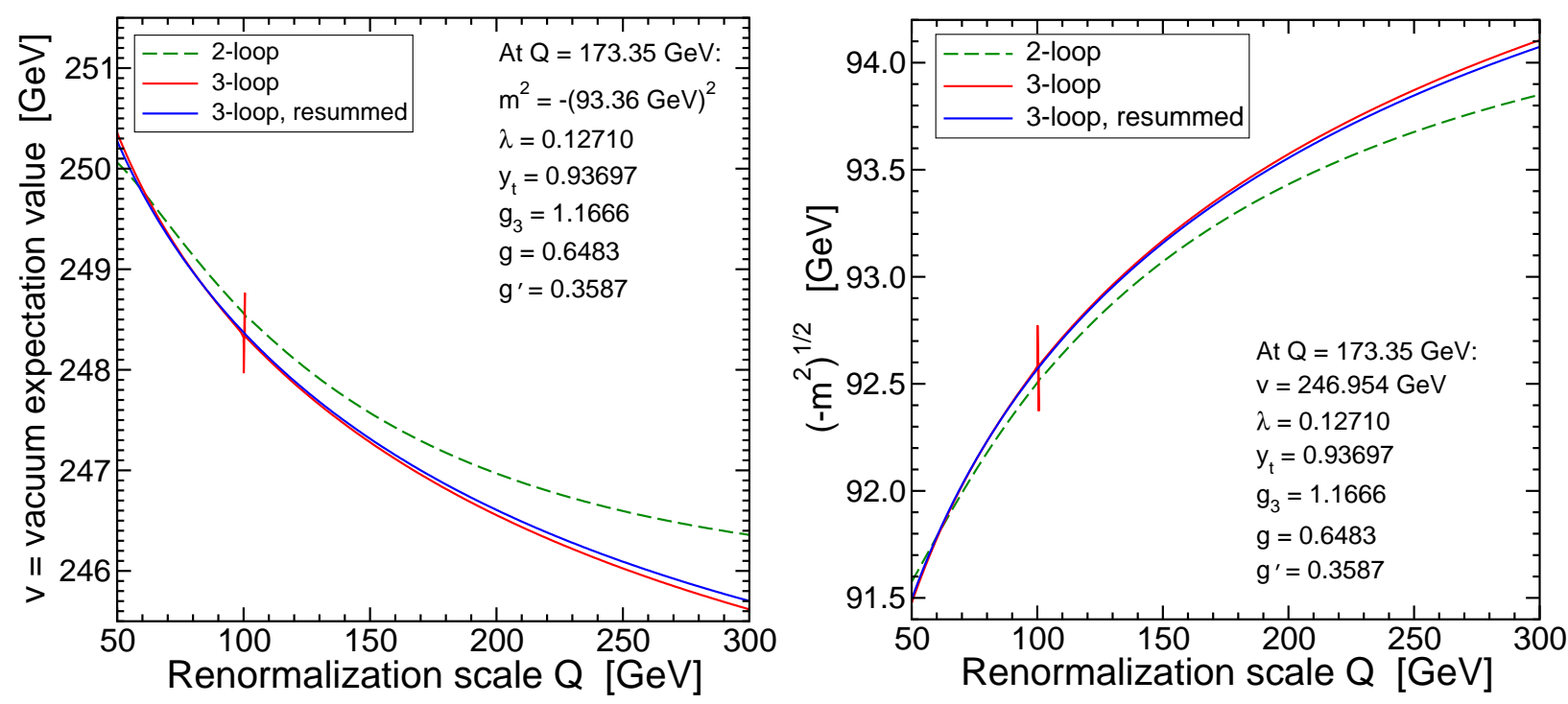

FIG. 5.1: Dependence of the VEV $v$ (left panel) and the Higgs Lagrangian mass parameter $\sqrt{-m^{2}}$ (right panel), as a function of the renormalization scale, as computed from the effective potential minimization condition at 2-loop order from ref. [29], at partial 3-loop order including also [31], and 3-loop order after resummation using eqs. (4.18)-(4.21). The input parameters are specified in eqs. (1.13)-(1.18) at the input scale $Q=173.35 \mathrm{GeV}$. In the left panel, the parameters including $m^{2}$ are run from the input scale to $Q$, and $v$ is solved for. In the right panel, the parameters (including $v=246.954 \mathrm{GeV}$ at $Q=173.35 \mathrm{GeV}$ ) are run to $Q$, and $m^{2}$ is solved for. The 3-loop case without resummation has a numerical instability associated with a failure of the iterative solution process to converge, represented by the vertical line of arbitrary height, for a narrow range near $Q=100.4$ $\mathrm{GeV}$, due to the $1 / G$ term in the minimization condition.

represented by a vertical line in the figure, for a range of several hundred $\mathrm{MeV}$ in $Q$ near $Q=100.4 \mathrm{GeV}$. (The height of this line in the figure is arbitrary, as no iterative solution to the minimization condition is obtained.) For other values of $Q$, the 3-loop result for $v$ is lower than the 2-loop result by up to a few hundred $\mathrm{MeV}$, depending on $Q$. The resummed 3-loop result does not differ much from the non-resummed result, except for the numerical instability region just mentioned. There, the resummed result of course remains perfectly smooth, as it does not depend on $G$ at all.

The right panel of Figure 5.1 shows the result of running the same parameters but $v$ instead of $m^{2}$, starting again from the input scale at $173.35 \mathrm{GeV}$, and then solving for $m^{2}$ at the scale $Q$ using eqs. (4.18)-(4.21). Again the difference between the usual 3-loop and resummed 3-loop calculations is very small except near the scale $Q=100.4 \mathrm{GeV}$ where $G$ goes through 0 , where the iterative process of solution again fails for the non-resummed case. 


\section{OUTLOOK}

In this paper, I have shown how issues of principle associated with the Goldstone boson contributions to the effective potential can be resolved through resummation. The minimization condition of eqs. (4.18)-(4.21), and the value of the effective potential at its minimum eqs. (4.28)-(4.32), do not involve $G$ at all, and so are manifestly free of spurious imaginary parts associated with $\ln (G)$ when $G$ is negative, and of divergences as $G \rightarrow 0$.

Given the tiny numerical impact found in the previous section for almost all renormalization scales, one might ask whether in practice one could not just use the usual prescription of minimizing the real part of the effective potential, taking care to choose a $Q$ to ensure that $G$ is not too close to 0. Here, I note that the minimization conditions eqs. (4.18)-(4.21) are actually easier to implement in practice, because there are fewer and less complicated terms and no need to deal with imaginary parts. The resummation method described above is also a useful ingredient in the analytical 2-loop calculation of the Standard Model Higgs mass. Both the resummed version of the minimization condition eqs. (4.18)-(4.21) and the 2-loop Higgs mass in the Standard Model will be implemented in a forthcoming public computer code [57].

A similar resummation of Goldstone contributions can clearly be applied to other cases of symmetry breaking beyond the Standard Model. For supersymmetry, the second derivatives of $V_{\text {eff }}$ have been used in one of the methods for approximating the lightest Higgs boson mass. The effective potential in minimal supersymmetry has the same behavior [58, 59] with respect to the tree-level squared masses of the Goldstone bosons at 2-loop order (except that $m_{G^{ \pm}}^{2}$ and $m_{G^{0}}^{2}$ are slightly different from each other when not at the minimum of the tree-level potential). The use of second derivatives, rather than first derivatives as in the minimization condition, means that the numerical instabilities associated with choices of renormalization

scale where $m_{G^{ \pm}}^{2} \approx 0$ and $m_{G^{0}}^{2} \approx 0$ can be much more significant; see Figure 1 in ref. [59] and the surrounding discussion. Resummation of the leading Goldstone contributions may also be useful in that case, although the ultimate resolution will come from calculating the full self-energy functions at non-zero external momentum invariant.

Note added: a paper [60] with significant overlap appeared in the same arXiv announcement as this one. 


\section{Appendix: Effective potential results in the Standard Model}

The 2-loop contribution to the Landau gauge Standard Model effective potential was found in ref. [29]. The result is:

$$
V_{2}=V_{S S S}+V_{S S}+V_{F F S}+V_{S S V}+V_{V V S}+V_{V S}+V_{F F V}+V_{\text {gauge }}
$$

where

$$
\begin{aligned}
& V_{S S S}=-3 \lambda^{2} v^{2}[I(H, H, H)+I(H, G, G)] \text {, } \\
& V_{S S}=\frac{3}{4} \lambda\left[A(H)^{2}+2 A(H) A(G)+5 A(G)^{2}\right] \text {, } \\
& V_{F F S}=3 y_{t}^{2}\left[(2 t-H / 2) I(H, t, t)-\frac{G}{2} I(G, t, t)+(t-G) I(0, G, t)\right. \\
& \left.+A(t)^{2}-A(H) A(t)-2 A(G) A(t)\right], \\
& V_{S S V}=\frac{g^{2}+g^{\prime 2}}{8} f_{S S V}(G, H, Z)+\frac{\left(g^{2}-g^{\prime 2}\right)^{2}}{8\left(g^{2}+g^{\prime 2}\right)} f_{S S V}(G, G, Z) \\
& +\frac{g^{2} g^{\prime 2}}{2\left(g^{2}+g^{\prime 2}\right)} f_{S S V}(G, G, 0)+\frac{g^{2}}{4}\left[f_{S S V}(G, H, W)+f_{S S V}(G, G, W)\right], \\
& V_{V V S}=\frac{g^{2} g^{\prime 2} v^{2}}{4\left(g^{2}+g^{\prime 2}\right)}\left[g^{\prime 2} f_{V V S}(W, Z, G)+g^{2} f_{V V S}(0, W, G)\right] \\
& +\frac{g^{4} v^{2}}{8} f_{V V S}(W, W, H)+\frac{\left(g^{2}+g^{\prime 2}\right)^{2} v^{2}}{16} f_{V V S}(Z, Z, H), \\
& V_{V S}=\frac{\left(g^{2}-g^{\prime 2}\right)^{2}}{4\left(g^{2}+g^{\prime 2}\right)} f_{V S}(Z, G)+\frac{g^{2}+g^{\prime 2}}{8}\left[f_{V S}(Z, H)+f_{V S}(Z, G)\right] \\
& +\frac{g^{2}}{4}\left[f_{V S}(W, H)+3 f_{V S}(W, G)\right] \\
& V_{F F V}=-\left[4 g_{3}^{2}+\frac{4 g^{2} g^{\prime 2}}{3\left(g^{2}+g^{\prime 2}\right)}\right] t f_{\overline{F F V}}(t, t, 0)+\frac{3 g^{2}}{2}\left[f_{F F V}(0, t, W)+3 f_{F F V}(0,0, W)\right] \\
& +\frac{1}{24\left(g^{2}+g^{\prime 2}\right)}\left[\left(9 g^{4}-6 g^{2} g^{\prime 2}+17 g^{\prime 4}\right) f_{F F V}(t, t, Z)\right. \\
& \left.+8 g^{2}\left(3 g^{2}-g^{\prime 2}\right) t f_{\overline{F F} V}(t, t, Z)+\left(63 g^{4}+6 g^{2} g^{\prime 2}+103 g^{4}\right) f_{F F V}(0,0, Z)\right], \\
& V_{\text {gauge }}=\frac{g^{2} g^{\prime 2}}{2\left(g^{2}+g^{\prime 2}\right)} f_{\text {gauge }}(W, W, 0)+\frac{g^{4}}{2\left(g^{2}+g^{\prime 2}\right)} f_{\text {gauge }}(W, W, Z)
\end{aligned}
$$

Here the loop functions are written in terms of the 1-loop function $A(x)$ defined in eq. (2.4) and a 2-loop function $I(x, y, z)$, which is invariant under interchange of any pair of $x, y, z$. It is equal to the $\epsilon$-independent part of the function $\left(16 \pi^{2}\right)^{2} \hat{I}(x, y, z)$ defined in ref. [29], and it is also given in terms of dilogarithms in eq. (2.19) of [30], and defined in section 2 of [49], 
which also provides a public computer code that evaluates it efficiently. The special cases of the functions $f_{S S V}, f_{V V S}, f_{V S}, f_{F F V}, f_{\overline{F F} V}$, and $f_{\text {gauge }}$ defined in ref. [30] that are pertinent for the Standard Model are:

$$
\begin{aligned}
& f_{S S V}(x, y, z)=\left[-\left(x^{2}+y^{2}+z^{2}-2 x y-2 x z-2 y z\right) I(x, y, z)+(x-y)^{2} I(0, x, y)\right. \\
& +(x-y-z) A(y) A(z)+(y-x-z) A(x) A(z)] / z \\
& +A(x) A(y)+2(x+y-z / 3) A(z), \\
& f_{S S V}(x, x, 0)=-3 A(x)^{2}+8 x A(x)-8 x^{2}, \\
& f_{V V S}(x, y, z)=\left[-\left(x^{2}+y^{2}+z^{2}+10 x y-2 x z-2 y z\right) I(x, y, z)\right. \\
& +(x-z)^{2} I(0, x, z)+(y-z)^{2} I(0, y, z)-z^{2} I(0,0, z) \\
& +y A(x) A(z)+x A(y) A(z)+(z-x-y) A(x) A(y)] / 4 x y \\
& +A(x) / 2+A(y) / 2+2 A(z)-x-y-z, \\
& f_{V V S}(0, x, y)=[(3 y-9 x) I(0, x, y)-3 y I(0,0, y)+3 A(x) A(y)] / 4 x \\
& +2 A(y)-3 x / 4-y / 2, \\
& f_{V S}(x, y)=3 A(x) A(y)+2 x A(y), \\
& f_{F F V}(x, x, 0)=0, \\
& f_{\overline{F F} V}(x, x, 0)=4 A(x)-8 x-6 A(x)^{2} / x, \\
& f_{F F V}(0, x, y)=\left[(x-y)(x+2 y) I(0, x, y)-x^{2} I(0,0, x)+(x-2 y) A(x) A(y)\right] / y \\
& +(2 y / 3-2 x) A(y)-2 x A(x)+x^{2}-y^{2}, \\
& f_{F F V}(x, x, y)=2(x-y) I(x, x, y)+2 A(x)^{2}-4 A(x) A(y)-4 x A(x) \\
& +(2 y / 3-4 x) A(y)+4 x^{2}-y^{2}, \\
& f_{\overline{F F} V}(x, x, y)=6 I(x, x, y)-8 A(x)+4 x+2 y, \\
& f_{\text {gauge }}(x, x, y)=(4 x-y)\left(12 x^{2}+20 x y+y^{2}\right) I(x, x, y) / 4 x^{2} \\
& +(x-y)^{2}\left(x^{2}+10 x y+y^{2}\right) I(0, x, y) / 2 x^{2} y \\
& +y\left(2 x^{2}-y^{2}\right) I(0,0, y) / 4 x^{2}+x(2 y-x) I(0,0, x) / 2 y \\
& +\left[y^{2}+18 x y-4 x^{2}\right] A(x)^{2} / 4 x^{2}+\left[x^{2}+23 x y-9 y^{2}\right] A(x) A(y) / 2 x y \\
& +[11 y+25 x / 3] A(x)+[11 x-4 y / 3] A(y)+14 x^{2}+24 x y+y^{2}, \\
& f_{\text {gauge }}(x, x, 0)=\frac{9 x}{2} I(0,0, x)-13 A(x)^{2}+\frac{100 x}{3} A(x)-\frac{21}{2} x^{2} \text {. }
\end{aligned}
$$

In this paper, the explicit form of $I(x, y, z)$ is not needed; instead, the calculations rely on several identities that it satisfies. First, we have

$$
I(0, x, x)=2 A(x)-2 x-A(x)^{2} / x
$$


which has been used in writing the equations above. Derivatives with respect to squared mass arguments are

$$
\begin{aligned}
\frac{\partial}{\partial x} I(x, y, z)= & {[(x-y-z) I(x, y, z)-2 A(y) A(z)+(x-y+z) A(x) A(y) / x} \\
& +(x+y-z) A(x) A(z) / x+(y+z-x)[A(x)+A(y)+A(z)] \\
& \left.+x^{2}-(y+z)^{2}\right] /\left(x^{2}+y^{2}+z^{2}-2 x y-2 x z-2 y z\right), \\
\frac{\partial}{\partial x} I(0, x, x)= & -A(x)^{2} / x^{2} .
\end{aligned}
$$

The derivative with respect to the renormalization scale $Q$ is:

$$
Q \frac{\partial}{\partial Q} I(x, y, z)=2 A(x)+2 A(y)+2 A(z)-2 x-2 y-2 z
$$

For making expansions in small $G$, the following results from eqs. (2.29)-(2.31) of ref. [30] are useful:

$$
\begin{aligned}
I(0,0, G)= & G\left[-\frac{1}{2} \overline{\ln }^{2}(G)+2 \overline{\ln }(G)-\frac{5}{2}-\frac{\pi^{2}}{6}\right] \\
I(G, G, x)= & I(0,0, x)+2 G[-x-I(0,0, x)+3 A(x)-A(x) \overline{\ln }(G)] / x+\mathcal{O}\left(G^{2}\right), \\
I(G, x, y)= & I(0, x, y)+G[-(x+y) I(0, x, y)-2 A(x) A(y) \\
& +3 x A(x)+3 y A(y)-y A(x)-x A(y)-(x+y)^{2} \\
& +(x-y)[A(y)-A(x)] \overline{\ln }(G)] /(x-y)^{2}+\mathcal{O}\left(G^{2}\right), \\
I(G, x, x)= & 2 A(x)-2 x-A(x)^{2} / x+G\left[4+A(x)^{2} /\left(2 x^{2}\right)+3 A(x) / x\right. \\
& -[1+A(x) / x] \overline{\ln }(G)]+\mathcal{O}\left(G^{2}\right) .
\end{aligned}
$$

The 3-loop contribution to the Standard Model effective potential, in the approximation $g_{3}, y_{t} \gg \lambda, g, g^{\prime}$, was found in ref. [31] (where it was written slightly differently):

$$
\begin{aligned}
V_{3}= & g_{3}^{4} t^{2}\left\{-184 \overline{\ln }^{3}(t)+868 \overline{\ln }^{2}(t)+\left(32 \zeta(3)-\frac{5642}{3}\right) \overline{\ln }(t)+\frac{16633}{9}\right. \\
& \left.+32 \zeta(3)+\frac{176}{135} \pi^{4}+\frac{64}{9} \ln ^{2}(2)\left[\pi^{2}-\ln ^{2}(2)\right]-\frac{512}{3} \operatorname{Li}_{4}(1 / 2)\right\} \\
& +g_{3}^{2} y_{t}^{2} t^{2}\left\{60 \overline{\ln }^{3}(t)-360 \overline{\ln }^{2}(t)+\left[814+12 \pi^{2}+240 \zeta(3)\right] \overline{\ln }(t)-\frac{2393}{3}\right. \\
& \left.-\frac{58}{3} \pi^{2}-216 \zeta(3)+\frac{124}{15} \pi^{4}+\frac{128}{3} \ln ^{2}(2)\left[\pi^{2}-\ln ^{2}(2)\right]-1024 \operatorname{Li}_{4}(1 / 2)\right\} \\
& +y_{t}^{4} t^{2}\left\{-\frac{333}{4} \overline{\ln }^{3}(t)+[189+81 \overline{\ln }(H)+27 \overline{\ln }(G)] \overline{\ln }^{2}(t)\right.
\end{aligned}
$$




$$
\begin{aligned}
& +\left[-\frac{6945}{8}-\frac{39}{4} \pi^{2}-36 \zeta(3)-54 \overline{\ln }(H)-54 \overline{\ln }(G)\right] \overline{\ln }(t) \\
& +\frac{15767}{16}+\frac{25}{2} \pi^{2}-\frac{22}{15} \pi^{4}-\frac{873}{2} \zeta(3)-8 \ln ^{2}(2)\left[\pi^{2}-\ln ^{2}(2)\right] \\
& \left.+192 \operatorname{Li}_{4}(1 / 2)+9 \overline{\ln }(H)+27 \overline{\ln }(G)\right\}
\end{aligned}
$$

or, numerically,

$$
\begin{aligned}
V_{3} \approx & g_{3}^{4} t^{2}\left\{-184 \overline{\ln }^{3}(t)+868 \overline{\ln }^{2}(t)-1842.20 \overline{\ln }(t)+1957.33\right\} \\
& +g_{3}^{2} y_{t}^{2} t^{2}\left\{60 \overline{\ln }^{3}(t)-360 \overline{\ln }^{2}(t)+1220.93 \overline{\ln }(t)-780.30\right\} \\
& +y_{t}^{4} t^{2}\left\{-83.25 \overline{\ln }^{3}(t)+[189+81 \overline{\ln }(H)+27 \overline{\ln }(G)] \overline{\ln }^{2}(t)\right. \\
& +[-1007.63-54 \overline{\ln }(H)-54 \overline{\ln }(G)] \overline{\ln }(t)+504.51+9 \overline{\ln }(H)+27 \overline{\ln }(G)\}
\end{aligned}
$$

For the check of renormalization group invariance mentioned at the end of section IV, the scalar field anomalous dimension and beta functions are

$$
\begin{aligned}
& \gamma_{\phi}=-Q \frac{d \ln \phi}{d Q}=\sum_{\ell=1}^{\infty} \frac{1}{\left(16 \pi^{2}\right)^{\ell}} \gamma_{\phi}^{(\ell)} \\
& \beta_{X}=Q \frac{d X}{d Q}=\sum_{\ell=1}^{\infty} \frac{1}{\left(16 \pi^{2}\right)^{\ell}} \beta_{X}^{(\ell)}
\end{aligned}
$$

with the 1-loop contributions:

$$
\begin{aligned}
& \gamma_{\phi}^{(1)}=3 y_{t}^{2}-9 g^{2} / 4-3 g^{\prime 2} / 4 \\
& \beta_{\lambda}^{(1)}=-6 y_{t}^{4}+12 \lambda y_{t}^{2}+24 \lambda^{2}-9 \lambda g^{2}-3 \lambda g^{2}+9 g^{4} / 8+3 g^{2} g^{2} / 4+3 g^{4} / 8 \\
& \beta_{m^{2}}^{(1)}=m^{2}\left[6 y_{t}^{2}+12 \lambda-9 g^{2} / 2-3 g^{2} / 2\right] \\
& \beta_{y_{t}}^{(1)}=y_{t}\left[9 y_{t}^{2} / 2-8 g_{3}^{2}-9 g^{2} / 4-17 g^{2} / 12\right] \\
& \beta_{g_{3}}^{(1)}=-7 g_{3}^{3} \\
& \beta_{g}^{(1)}=-19 g^{3} / 6 \\
& \beta_{g^{\prime}}^{(1)}=41 g^{\prime 3} / 6 .
\end{aligned}
$$

The necessary 2-loop contributions are [50 53], [29]:

$$
\begin{aligned}
\gamma_{\phi}^{(2)}= & 20 g_{3}^{2} y_{t}^{2}-27 y_{t}^{4} / 4+45 y_{t}^{2} g^{2} / 8+85 y_{t}^{2} g^{\prime 2} / 24+6 \lambda^{2}-271 g^{4} / 32 \\
& +9 g^{2} g^{\prime 2} / 16+431 g^{\prime 4} / 96
\end{aligned}
$$




$$
\begin{aligned}
\beta_{\lambda}^{(2)}= & -32 g_{3}^{2} y_{t}^{4}+30 y_{t}^{6}-8 y_{t}^{4} g^{\prime 2} / 3-3 \lambda y_{t}^{4}+80 g_{3}^{2} y_{t}^{2} \lambda-144 y_{t}^{2} \lambda^{2}+45 y_{t}^{2} \lambda g^{2} / 2 \\
& +85 y_{t}^{2} \lambda g^{\prime 2} / 6-9 y_{t}^{2} g^{4} / 4+21 y_{t}^{2} g^{2} g^{\prime 2} / 2-19 y_{t}^{2} g^{4} / 4-312 \lambda^{3}+108 \lambda^{2} g^{2} \\
& +36 \lambda^{2} g^{\prime 2}-73 \lambda g^{4} / 8+39 \lambda g^{2} g^{\prime 2} / 4+629 \lambda g^{\prime 4} / 24+305 g^{6} / 16 \\
& -289 g^{4} g^{\prime 2} / 48-559 g^{2} g^{\prime 4} / 48-379 g^{\prime 6} / 48, \\
\beta_{m^{2}}^{(2)}= & m^{2}\left[40 g_{3}^{2} y_{t}^{2}-27 y_{t}^{4} / 2-72 \lambda y_{t}^{2}+45 y_{t}^{2} g^{2} / 4+85 y_{t}^{2} g^{\prime 2} / 12-60 \lambda^{2}\right. \\
& \left.+72 \lambda g^{2}+24 \lambda g^{\prime 2}-145 g^{4} / 16+15 g^{2} g^{\prime 2} / 8+557 g^{\prime 4} / 48\right], \\
\beta_{y_{t}}^{(2)}= & y_{t}\left[-108 g_{3}^{4}+36 g_{3}^{2} y_{t}^{2}-12 y_{t}^{4}+\ldots\right] .
\end{aligned}
$$

Partial 3-loop contributions are needed here only for the beta function for $\lambda[54-56]$ :

$$
\beta_{\lambda}^{(3)}=g_{3}^{4} y_{t}^{4}[64 \zeta(3)-532 / 3]+g_{3}^{2} y_{t}^{6}[480 \zeta(3)-76]+y_{t}^{8}[-72 \zeta(3)-1599 / 4]+\ldots
$$

Finally, the beta function contributions for the field-independent vacuum energy density are:

$$
\begin{aligned}
& \beta_{\Lambda}^{(1)}=2\left(m^{2}\right)^{2}, \\
& \beta_{\Lambda}^{(2)}=\left(12 g^{2}+4 g^{2}-12 y_{t}^{2}\right)\left(m^{2}\right)^{2} .
\end{aligned}
$$

Acknowledgments: I thank Hiren Patel for discussions. This work was supported in part by the National Science Foundation grant number PHY-1068369.

[1] G. Aad et al. [ATLAS Collaboration], "Observation of a new particle in the search for the Standard Model Higgs boson with the ATLAS detector at the LHC," Phys. Lett. B 716, 1 (2012) [1207.7214],

[2] S. Chatrchyan et al. [CMS Collaboration], "Observation of a new boson at a mass of $125 \mathrm{GeV}$ with the CMS experiment at the LHC," Phys. Lett. B 716, 30 (2012) [1207.7235].

[3] [ATLAS Collaboration], "Combined measurements of the mass and signal strength of the Higgs-like boson with the ATLAS detector using up to $25 \mathrm{fb}^{-1}$ of proton-proton collision data," ATLAS-CONF-2013-014, March 6, 2013.

[4] [CMS Collaboration], "Combination of standard model Higgs boson searches and measurements of the properties of the new boson with a mass near 125 GeV" CMS-PAS-HIG-12-045, November 16, 2012.

[5] S. R. Coleman and E. J. Weinberg, "Radiative Corrections as the Origin of Spontaneous Symmetry Breaking," Phys. Rev. D 7, 1888 (1973).

[6] R. Jackiw, "Functional evaluation of the effective potential," Phys. Rev. D 9, 1686 (1974).

[7] M. Sher, "Electroweak Higgs Potentials and Vacuum Stability," Phys. Rept. 179, 273 (1989), and references therein.

[8] M. Lindner, M. Sher and H. W. Zaglauer, "Probing Vacuum Stability Bounds at the Fermilab Collider," Phys. Lett. B 228, 139 (1989).

[9] P. B. Arnold and S. Vokos, "Instability of hot electroweak theory: bounds on m(H) and M(t)," Phys. Rev. D 44, 3620 (1991).

[10] C. Ford, D. R. T. Jones, P. W. Stephenson and M. B. Einhorn, "The Effective potential and 
the renormalization group," Nucl. Phys. B 395, 17 (1993) hep-lat/9210033.

[11] J. A. Casas, J. R. Espinosa and M. Quirós, "Improved Higgs mass stability bound in the standard model and implications for supersymmetry," Phys. Lett. B 342, 171 (1995) hep-ph/9409458.

[12] J. R. Espinosa and M. Quiros, "Improved metastability bounds on the standard model Higgs mass," Phys. Lett. B 353, 257 (1995) hep-ph/9504241].

[13] J. A. Casas, J. R. Espinosa and M. Quiros, "Standard model stability bounds for new physics within LHC reach," Phys. Lett. B 382 (1996) 374 [hep-ph/9603227].

[14] G. Isidori, G. Ridolfi and A. Strumia, "On the metastability of the standard model vacuum," Nucl. Phys. B 609, 387 (2001) hep-ph/0104016.

[15] J. R. Espinosa, G. F. Giudice and A. Riotto, "Cosmological implications of the Higgs mass measurement," JCAP 0805, 002 (2008) [0710.2484].

[16] N. Arkani-Hamed, S. Dubovsky, L. Senatore and G. Villadoro, "(No) Eternal Inflation and Precision Higgs Physics," JHEP 0803, 075 (2008) [0801.2399].

[17] F. Bezrukov and M. Shaposhnikov, "Standard Model Higgs boson mass from inflation: Two loop analysis," JHEP 0907, 089 (2009) [0904.1537].

[18] J. Ellis, J. R. Espinosa, G. F. Giudice, A. Hoecker and A. Riotto, "The Probable Fate of the Standard Model," Phys. Lett. B 679, 369 (2009) [0906.0954].

[19] J. Elias-Miro, J. R. Espinosa, G. F. Giudice, G. Isidori, A. Riotto and A. Strumia, "Higgs mass implications on the stability of the electroweak vacuum," Phys. Lett. B 709, 222 (2012) [1112.3022].

[20] S. Alekhin, A. Djouadi and S. Moch, "The top quark and Higgs boson masses and the stability of the electroweak vacuum," Phys. Lett. B 716, 214 (2012) [1207.0980].

[21] F. Bezrukov, M. Y. Kalmykov, B. A. Kniehl and M. Shaposhnikov, "Higgs Boson Mass and New Physics," JHEP 1210, 140 (2012) [1205.2893].

[22] G. Degrassi, S. Di Vita, J. Elias-Miro, J. R. Espinosa, G. F. Giudice, G. Isidori and A. Strumia, "Higgs mass and vacuum stability in the Standard Model at NNLO," JHEP 1208, 098 (2012) [1205.6497].

[23] D. Buttazzo, G. Degrassi, P. P. Giardino, G. F. Giudice, F. Sala, A. Salvio and A. Strumia, "Investigating the near-criticality of the Higgs boson," [1307.3536].

[24] H. Yamagishi, "Coupling Constant Flows and Dynamical Symmetry Breaking," Phys. Rev. D 23, 1880 (1981). H. Yamagishi, "Renormalization Group Analysis of Supersymmetric Mass Hierarchies," Nucl. Phys. B 216, 508 (1983).

[25] M. B. Einhorn and D. R. T. Jones, "Scale Fixing by Dimensional Transmutation: Supersymmetric Unified Models and the Renormalization Group," Nucl. Phys. B 211, 29 (1983).

[26] B. M. Kastening, "Renormalization group improvement of the effective potential in massive phi**4 theory," Phys. Lett. B 283, 287 (1992).

[27] M. Bando, T. Kugo, N. Maekawa and H. Nakano, "Improving the effective potential," Phys. Lett. B 301, 83 (1993) hep-ph/9210228. "Improving the effective potential: Multimass scale case," Prog. Theor. Phys. 90, 405 (1993) hep-ph/9210229].

[28] M. B. Einhorn and D. R. T. Jones, "The Effective potential, the renormalisation group and vacuum stability," JHEP 0704, 051 (2007) hep-ph/0702295].

[29] C. Ford, I. Jack and D.R.T. Jones, "The Standard model effective potential at two loops," Nucl. Phys. B 387, 373 (1992) [Erratum-ibid. B 504, 551 (1997)] [hep-ph/0111190].

[30] S.P. Martin, "Two loop effective potential for a general renormalizable theory and softly broken supersymmetry," Phys. Rev. D 65, 116003 (2002) hep-ph/0111209.

[31] S.P. Martin, "Three-loop Standard Model effective potential at leading order in strong and top Yukawa couplings," Phys. Rev. D 89, 013003 (2014) arXiv:1310.7553 [hep-ph]].

[32] L. Dolan and R. Jackiw, "Gauge Invariant Signal for Gauge Symmetry Breaking," Phys. Rev. D 9, 2904 (1974).

[33] J. S. Kang, "Gauge Invariance of the Scalar-Vector Mass Ratio in the Coleman-Weinberg Model," Phys. Rev. D 10, 3455 (1974).

[34] W. Fischler and R. Brout, "Gauge Invariance in Spontaneously Broken Symmetry," Phys. Rev. D 11, 905 (1975). 
[35] J. -M. Frere and P. Nicoletopoulos, "Gauge Invariant Content of the Effective Potential," Phys. Rev. D 11, 2332 (1975).

[36] N. K. Nielsen, "On the Gauge Dependence of Spontaneous Symmetry Breaking in Gauge Theories," Nucl. Phys. B 101, 173 (1975).

[37] R. Fukuda and T. Kugo, "Gauge Invariance in the Effective Action and Potential," Phys. Rev. D 13, 3469 (1976).

[38] I. J. R. Aitchison and C. M. Fraser, "Gauge Invariance and the Effective Potential," Annals Phys. 156, 1 (1984).

[39] R. Kobes, G. Kunstatter and A. Rebhan, "Gauge dependence identities and their application at finite temperature," Nucl. Phys. B 355, 1 (1991).

[40] D. Metaxas and E. J. Weinberg, "Gauge independence of the bubble nucleation rate in theories with radiative symmetry breaking," Phys. Rev. D 53, 836 (1996) hep-ph/9507381.

[41] P. Gambino and P. A. Grassi, "The Nielsen identities of the SM and the definition of mass," Phys. Rev. D 62, 076002 (2000) hep-ph/9907254.

[42] L. P. Alexander and A. Pilaftsis, "The One-Loop Effective Potential in Non-Linear Gauges," J. Phys. G 36, 045006 (2009) arXiv:0809.1580 [hep-ph]].

[43] W. Loinaz and R. S. Willey, "Gauge dependence of lower bounds on the Higgs mass derived from electroweak vacuum stability constraints," Phys. Rev. D 56, 7416 (1997) hep-ph/9702321.

[44] O. M. Del Cima, D. H. T. Franco and O. Piguet, "Gauge independence of the effective potential revisited," Nucl. Phys. B 551, 813 (1999) hep-th/9902084.

[45] H. H. Patel and M. J. Ramsey-Musolf, "Baryon Washout, Electroweak Phase Transition, and Perturbation Theory," JHEP 1107, 029 (2011) arXiv:1101.4665 [hep-ph]].

[46] L. Di Luzio and L. Mihaila, "On the gauge dependence of the Standard Model vacuum instability scale," arXiv:1404.7450 [hep-ph].

[47] N. K. Nielsen, "Removing the gauge parameter dependence of the effective potential by a field redefinition," arXiv:1406.0788 [hep-ph].

[48] E. J. Weinberg and A. -q. Wu, "Understanding Complex Perturbative Effective Potentials," Phys. Rev. D 36, 2474 (1987).

[49] S. P. Martin and D. G. Robertson, "TSIL: A Program for the calculation of two-loop selfenergy integrals," Comput. Phys. Commun. 174, 133 (2006) hep-ph/0501132].

[50] M. E. Machacek and M. T. Vaughn, "Two Loop Renormalization Group Equations in a General Quantum Field Theory. 1. Wave Function Renormalization," Nucl. Phys. B 222, 83 (1983).

[51] M. E. Machacek and M. T. Vaughn, "Two Loop Renormalization Group Equations in a General Quantum Field Theory. 2. Yukawa Couplings," Nucl. Phys. B 236, 221 (1984).

[52] I. Jack and H. Osborn, "General Background Field Calculations With Fermion Fields," Nucl. Phys. B 249, 472 (1985).

[53] M. E. Machacek and M. T. Vaughn, "Two Loop Renormalization Group Equations in a General Quantum Field Theory. 3. Scalar Quartic Couplings," Nucl. Phys. B 249, 70 (1985).

[54] K. G. Chetyrkin and M. F. Zoller, "Three-loop $\beta$-functions for top-Yukawa and the Higgs self-interaction in the Standard Model," JHEP 1206, 033 (2012) [1205.2892].

[55] K. G. Chetyrkin and M. F. Zoller, " $\beta$-function for the Higgs self-interaction in the Standard Model at three-loop level," JHEP 1304, 091 (2013) [1303.2890].

[56] A. V. Bednyakov, A. F. Pikelner and V. N. Velizhanin, "Higgs self-coupling beta-function in the Standard Model at three loops," Nucl. Phys. B 875, 552 (2013) [1303.4364].

[57] S. P. Martin and D. G. Robertson, to appear.

[58] S. P. Martin, "Two loop effective potential for the minimal supersymmetric standard model," Phys. Rev. D 66, 096001 (2002) [hep-ph/0206136].

[59] S. P. Martin, "Complete two loop effective potential approximation to the lightest Higgs scalar boson mass in supersymmetry," Phys. Rev. D 67, 095012 (2003) hep-ph/0211366.

[60] J. Elias-Miro, J. R. Espinosa and T. Konstandin, "Taming Infrared Divergences in the Effective Potential," arXiv:1406.2652 [hep-ph]. 